\title{
Eribulin in the management of inoperable soft- tissue sarcoma: patient selection and survival
}

This article was published in the following Dove Press journal:

OncoTargets and Therapy

9 September 2016

Number of times this article has been viewed

\section{Colin Thomas \\ Sujana Movva}

Department of Hematology/ Oncology, Fox Chase Cancer Center, Philadelphia, PA, USA
Correspondence: Sujana Movva Department of Hematology/Oncology, Fox Chase Cancer Center, 333 Cottman Avenue, Philadelphia, PA I9III, USA Email sujana.movva@fccc.edu

\begin{abstract}
Patients diagnosed with metastatic soft-tissue sarcoma (STS) have a poor prognosis. Additionally, after failure of first-line therapy, there are relatively few treatment options from which to choose. The novel tubulin-binding drug, eribulin, with a unique mechanism of action from taxanes or vinca alkaloids, has shown clinical activity in several different types of cancers. Eribulin has been approved by the US Food and Drug Administration (FDA) for patients with metastatic breast cancer previously treated with an anthracycline or a taxane and has recently been FDA approved for patients with unresectable or metastatic liposarcoma who have failed a previous anthracycline regimen. Here, we review current standard treatments of STS, a background of eribulin, the studies that have propelled eribulin to FDA approval for liposarcoma, and future directions of the drug. The benefits of eribulin in STS are discussed in detail, especially with regard to the recent pivotal Phase III study comparing eribulin to dacarbazine for leiomyosarcoma and adipocytic sarcoma.
\end{abstract}

Keywords: eribulin, tubulin-binding drugs, soft-tissue sarcoma

\section{Introduction: sarcoma}

Sarcomas are a rare presentation of cancer, comprising $<1 \%$ of adult malignancies and $\sim 12 \%$ of cancers in children. ${ }^{1-3}$ They derive from cells of mesenchymal origin, thereby producing cancers of a broad variety of histopathology. Sarcomas are further divided into soft-tissue sarcoma (STS) - where the histopathology includes muscle, adipose, cartilage, blood and lymph vessels, peripheral nerves, and fibrous tissue - and non-STS, involving bone. STS comprises $80 \%$ of all sarcomas. Additionally, in the US, there are $\sim 12,000$ new cases of STSs every year, resulting in almost 5,000 yearly deaths. ${ }^{3}$

The most life-threatening aspect of sarcomas is their ability to disseminate hematogenously. Where sarcomas metastasize, however, is dependent on the tumor type and primary location. ${ }^{4}$ Metastasized STS generally indicates a poor prognosis with a median survival ranging between 11 and 15 months but with newer median overall survival (OS) trends closer to 18 months., ${ }^{5,6}$ Treating metastatic STS, therefore, usually abides by the principle of palliation. While evaluating the efficacy of chemotherapy in metastatic STS, it has become increasingly clear that histologic subtype plays an important role. Because there are over 50 different histologic subtypes of STSs with various chemosensitivity profiles and prognoses, many studies assessing treatment options have been compromised by lumping all STSs into one category. ${ }^{2}$ Given the palliative nature of treatment for metastasized STS, assessing end points in treatment has been challenging. There appears to be a disparity between a sarcoma's objective response rate and the overall stability of the disease. Assessment of treatments therefore often uses OS and progression-free survival (PFS) as end points. Because of the 
importance in evaluating disease-stabilizing drugs for STS which may lack dramatic cytoreductive capabilities, baseline references on PFS have been determined from a clinical trials database for active and inactive STS regimens to be used in Phase II efficacy studies. ${ }^{7}$

Among all drug treatments for STS, the most dramatically successful has been the tyrosine kinase inhibitor, imatinib, in gastrointestinal stromal tumors (GISTs). Imatinib has demonstrated significant efficacy in GISTs in several clinical circumstances. ${ }^{8}$ With regard to non-GIST STS, the optimal chemotherapy depends on histology and goals of care. Single-agent therapies with activity include doxorubicin (ie, an anthracycline) and ifosfamide. ${ }^{9-11}$ For second-/ third-line chemotherapy, dacarbazine, an alkylating agent, has shown modest efficacy with comparable disease control to other second-/third-line agents such as ifosfamide but with more favorable side effects. ${ }^{12}$ A recent Phase III study, however, has shown superiority in PFS of trabectedin over dacarbazine in metastatic STS patients who already failed at least two prior regimens. ${ }^{13}$ The trabectedin study, which included 518 patients, found an improvement in median PFS of 4.2 versus 1.5 months in the dacarbazine arm $(P<0.001)$. The median OS, however, was not significantly improved at 12.4 versus 12.9 months in the trabectedin and dacarbazine arms, respectively $(P=0.37)$. Pazopanib, the multitargeted tyrosine kinase inhibitor, has also been shown to significantly extend PFS, but not OS, in patients who progressed on up to four lines of standard non-antiangiogenesis inhibitor chemotherapy. ${ }^{14}$ The Phase III pazopanib trial, which evaluated 369 patients, found a median PFS of 4.6 months with pazopanib versus 1.6 months in the placebo arm $(P<0.0001)$. The median OS was 12.5 versus 10.7 months in the pazopanib and placebo arms, respectively $(P=0.25)$.

Taxanes have also been shown to be efficacious as a single agent in advanced angiosarcoma. ${ }^{15}$ Other unique sensitivity profiles specific to certain histologic subtypes include myxoid liposarcomas, which tend to be more sensitive to doxorubicin or trabectedin, and synovial sarcoma, which tends to be more sensitive toward ifosfamide. ${ }^{16,17}$ Another widely used agent in STS is gemcitabine, which has been evaluated within several different contexts of STS. Overall, there has been activity of gemcitabine alone or with docetaxel as therapy in STS and specifically leiomyosarcoma. A Phase II study comparing gemcitabine alone with gemcitabine with docetaxel showed superiority of gemcitabine/docetaxel for patients with anywhere between zero and three prior treatment regimens. ${ }^{18}$ Additionally, a recent Phase II study demonstrated that gemcitabine with docetaxel had efficacy as first-line therapy in leiomyosarcoma. ${ }^{19}$
Most combination chemotherapy regimens for metastasized STS include doxorubicin in some variation. Common combinations include doxorubicin with ifosfamide, doxorubicin with dacarbazine, and doxorubicin with ifosfamide and dacarbazine. When comparing these combination regimens with the single-agent doxorubicin, however, there is no difference in OS despite there being a greater response rate and longer median PFS when using combination therapies. ${ }^{20,21}$ What is also important to note is that the combination therapies have significantly greater toxicity than doxorubicin alone. ${ }^{20}$

\section{Eribulin background Mode of action}

Eribulin's antitumor activity stems from its novel ability to disrupt microtubule polymerization, without affecting depolymerization, by binding a unique site on tubulin. It has the capacity to sequester tubulin dimers into aggregates that cannot be utilized by the cell. ${ }^{22,23}$ The irreversible inhibition of mitosis which eribulin produces from disrupting microtubule dynamics ultimately results in the arrest of cancer cell growth and eventual apoptosis via prometaphase blockage. ${ }^{24}$ Other than producing an arrest of mitosis, eribulin has also been shown to inhibit Wnt/ $\beta$-catenin signaling in small bowel adenocarcinoma cell line experiments as well as alter tumor vasculature resulting in greater perfusion and drug delivery. ${ }^{25,26}$ Tumor metastasis also seems to be affected by eribulin via reversing epithelial-mesenchymal transition. ${ }^{27}$

Preclinical and clinical studies of eribulin were accelerated after it was successfully synthesized from its parent marine sponge-derived compound, halichondrin B. ${ }^{28,29}$ Preclinically, eribulin was compared to the antimitotic drugs vinblastine and paclitaxel using in vitro cancer cell lines and in vivo xenografts. Eribulin was found to inhibit growth of several cancer lines at significantly lesser concentrations than vinblastine and paclitaxel while also producing less toxicity. ${ }^{23}$ In other mouse model experiments, eribulin was shown to produce less neurotoxicity than other microtubule-directed cancer drugs. ${ }^{30}$ Activity of eribulin was seen in the cell lines of breast, colon, non-small-cell lung, small-cell lung, prostate, ovarian, and head and neck cancers as well as uterine sarcoma, promyelocytic leukemia, histiocytic lymphoma, and melanoma. ${ }^{31}$

\section{Pharmacology}

Eribulin's action on microtubule dynamics and tubulin sequestration is concentration dependent. ${ }^{22}$ Although eribulin binds $\beta$-tubulin at a single site, the maximum stoichiometry is 15 molecules of eribulin per microtubule - demonstrating 
high binding affinity. ${ }^{32}$ Eribulin fits between two heterodimers next to the exchangeable-site nucleotide. ${ }^{33}$ The binding site of eribulin is only at the microtubule plus ends, a site different from that of other tubulin-binding drugs such as taxanes and vinca alkaloids. ${ }^{32}$ Further comparison of eribulin with other tubulin-binding drugs shows that both eribulin and vinca alkaloids prevent microtubule polymerization, thereby suppressing the microtubule growth phase, as well as preventing $\beta$-tubulin nucleotide exchange and cross-linking; however, the microtubule polymers formed by their pharmacologic stabilization differ between vinca alkaloids and eribulin, resulting in large stable polymers and smaller unstable polymers, respectively. ${ }^{33}$ Vinca alkaloids also aggregate tubulin into spirals, while eribulin aggregates tubulin into globular structures, overall highlighting that pharmacology of eribulin is different from that of other tubulin-binding drugs. ${ }^{33}$

\section{Phase I trials}

Several Phase I trials have evaluated eribulin in patients with advanced solid malignancies with regard to maximum tolerated dose (MTD) and dosing toxicity. Four Phase I studies determined MTDs ranging from 1 to $2 \mathrm{mg} / \mathrm{m}^{2}$ based on different cycles and infusions. In the first Phase I trial, eribulin was administered as a weekly bolus for 3 weeks out of 4 . Dose-limiting toxicities (DLTs) of febrile neutropenia and grade 4 neutropenia occurred at the $2.0 \mathrm{mg} / \mathrm{m}^{2} /$ week dose level. Therefore, $1.4 \mathrm{mg} / \mathrm{m}^{2} /$ week was established as the MTD. Responses were seen in two patients with non-smallcell lung cancer (NSCLC) and bladder cancer, and there were 15 patients with stable disease. ${ }^{34}$ Goel et al determined an MTD of $1 \mathrm{mg} / \mathrm{m}^{2}$, with maximal dosing to $1.4 \mathrm{mg} / \mathrm{m}^{2}$ given as an intravenous (IV) infusion over 1 hour on days 1,8 , and 15 of a 28-day cycle. Two out of the nine patients maximally dosed experienced a DLT of grade 4 neutropenia. Three additional patients experienced grade 3 neutropenia at this dose level, requiring dose omissions. ${ }^{35}$ Of the 32 patients in this trial, ten achieved stable disease, and one patient with cervical cancer had an unconfirmed partial response lasting several months. Tan et al demonstrated an MTD of eribulin of $2 \mathrm{mg} / \mathrm{m}^{2}$ infused over 1 hour every 21 days. Only one of seven patients at $2 \mathrm{mg} / \mathrm{m}^{2}$ experienced a neutropenic DLT with other adverse effects including fatigue, anorexia, nausea, and alopecia. ${ }^{36}$ Additionally, one patient, among the 21 evaluated in this study, with NSCLC had an unconfirmed partial response, while 12 patients achieved stable disease. Across the Phase I studies, DLTs were related to neutropenia and febrile neutropenia. Other adverse events were related to fatigue, anorexia, alopecia, and nausea. There was also a low incidence of severe neuropathy. The weekly bolus schedule was chosen for further development of eribulin. However, the subsequent Phase II study of eribulin given at $1.4 \mathrm{mg} / \mathrm{m}^{2}$ on days 1,15 , and 21 every 28 days had a high rate of dose delays (63\%) due to neutropenia on day 15 of the first cycle. ${ }^{37}$ Neutropenia on day 15 had not been included as a DLT in the first Phase I study of eribulin. A fourth Phase I study from Japan formally evaluated eribulin as 2- to 10-minute IV injections on days 1 and 8 of a 21-day cycle, in order to determine whether doses $>1.4 \mathrm{mg} / \mathrm{m}^{2}$ could be tolerated. Two of three patients and three of three patients dosed at 1.4 and $2.0 \mathrm{mg} / \mathrm{m}^{2}$, respectively, experienced DLTs of neutropenia and febrile neutropenia. Therefore, the authors recommended a dose of $1.4 \mathrm{mg} / \mathrm{m}^{2}$ on days 1 and 8 every 21 days for future trials. ${ }^{38}$

\section{Pharmacokinetics}

At the bolus weekly dose given for 3 weeks of 4, eribulin demonstrated a triphasic elimination and a prolonged halflife of 36-48 hours. At the MTD of $1.4 \mathrm{mg} / \mathrm{m}^{2}$, the concentration of eribulin was above that required for in vitro cytotoxicity for $>1$ week. ${ }^{34}$ When given as an infusion over an hour on the same schedule, there was linear pharmacokinetics, and the dose was proportional over the range of $0.25-1.4 \mathrm{mg} / \mathrm{m}^{2}$. The plasma concentration-time profile exhibited a rapid distribution phase with a mean distribution half-life of $\approx 0.43$ hours followed by a slower elimination phase with a half-life of 38.7 hours. ${ }^{35}$ Eribulin given over 1 hour every 3 weeks demonstrated an extensive volume of distribution and a slow-to-moderate clearance and a plasma terminal half-life of $\sim 2$ days. ${ }^{36}$ Minimal urinary excretion was seen across all studies. When eribulin was administered as a bolus at $1.4 \mathrm{mg} / \mathrm{m}^{2}$ on days 1 and 8 every 21 days, there was triphasic pharmacokinetics over the dosing range of $0.7-2.0 \mathrm{mg} / \mathrm{m}^{2}$. A long terminal half-life of 36.4-59.9 hours was again noted, with low systemic clearance and a high volume of distribution. ${ }^{38}$

\section{Eribulin in the treatment of cancer}

Outside of sarcoma, there have been three randomized Phase III trials evaluating eribulin: two in metastatic breast cancer and one in advanced NSCLC. The EMBRACE trial included patients who had either metastatic or locally recurrent breast cancer and who had already been treated with between two and five prior chemotherapies which included a taxane and an anthracycline. Eribulin $1.4 \mathrm{mg} / \mathrm{m}^{2}$ for 2-5 minutes on days 1 and 8 of a 21-day cycle was compared to treatment of physician's choice. The study demonstrated an OS of 2.5 months greater in the eribulin arm (13.1 versus 10.6 months; hazard ratio $[\mathrm{HR}]=0.81,95 \%$ confidence 
interval $[\mathrm{CI}]=0.66-0.99 ; P=0.041)$, with response rates of $12 \%$ versus $5 \%(P=0.002)$ in patients treated with eribulin versus physician's choice, respectively. ${ }^{39}$ There was no difference in PFS between the two groups. The results were confirmed in an updated analysis as requested by the European and the US regulatory authorities. The most common toxicities were fatigue, neutropenia, and asthenia. Five percent of patients had peripheral neuropathy leading to discontinuation of eribulin, with grade 3 or 4 neuropathy occurring in $8 \%$ of patients. The EMBRACE trial led to eribulin's US Food and Drug Administration (FDA) approval in 2010 for metastatic breast cancer in patients previously treated with an anthracycline or a taxane. A separate Phase III study of eribulin in metastatic breast cancer compared eribulin to capecitabine in patients treated with up to three prior chemotherapy agents. Patients were required to have been treated with an anthracycline and a taxane previously. There was no difference in OS, PFS, or response rate between the two drugs. ${ }^{40}$ This difference in outcome may be due to the impact of post-progression therapies as patients in the latter trial were exposed to eribulin earlier in their disease course than in the EMBRACE trial. When data from both Phase III trials were pooled, however, eribulin was found to improve OS in all patient subgroups including HER2-negative disease. ${ }^{41}$

In advanced NSCLC, eribulin was once again compared to physician's choice therapy. Patients were required to have been treated with at least two different chemotherapy regimens. Despite promising Phase II data, the Phase III trial did not demonstrate any significant difference in OS or PFS between the two treatment options. ${ }^{42}$ Although the Phase III data of eribulin in NSCLC were disappointing, efficacy of the drug in other cancers is being realized. Apart from the FDA approval of eribulin in metastatic breast cancer, there have been Phase II trials evaluating eribulin in ovarian, pancreatic, and metastatic castration-resistant prostate cancer. ${ }^{43-46}$

\section{Eribulin in sarcoma}

Eribulin has shown antitumor activity in preclinical models of fibrosarcoma and leiomyosarcoma xenografts. ${ }^{47}$ Eribulin was first evaluated clinically in STS in a non-randomized, multicenter Phase II trial. ${ }^{48}$ Patients enrolled were allowed to have previously received only one combination chemotherapy regimen or two single-drug regimens for their advanced disease. The study included 128 patients, stratified by subtype: leiomyosarcoma $(n=40)$, adipocytic sarcoma $(n=37)$, synovial sarcoma $(n=19)$, and others $(n=32)$. Eribulin was administered at a dose of $1.4 \mathrm{mg} / \mathrm{m}^{2}$ as 2- to 5-minute infusions on days 1 and 8 every 21 days. Eighty-nine percent of patients had received doxorubicin previously. The primary end point was PFS at 12 weeks after the start of therapy until disease progression or intolerance. Eribulin reached the predefined statistical targets of PFS in leiomyosarcoma and adipocytic sarcoma, with $31.6 \%$ and $46.9 \%$ of patients meeting the primary end point, respectively. ${ }^{48}$ Among the leiomyosarcoma patients, the rate of PFS was comparable irrespective of whether the tumor was derived from the female reproductive tract or not. Likewise, for the patients with adipocytic sarcomas, the statistics were comparable among dedifferentiated, pleomorphic, myxoid, round cell, and histological subtypes not otherwise specified. Best overall response as per Response Evaluation Criteria in Solid Tumors was $4.3 \%$ for the entire cohort, with one complete response in a patient with dedifferentiated liposarcoma.

Despite not meeting the statistical targets in the other subtypes of STSs, responses to eribulin treatment were seen, most notably in fibroblastic sarcoma, malignant solitary fibrous tumor, and epithelioid sarcoma - subtypes of sarcomas known for being relatively refractory to chemotherapy. The Phase II trial also demonstrated that when given at $1.4 \mathrm{mg} / \mathrm{m}^{2}$ as 2 - to 5 -minute IV infusions on days 1 and 8 of 21-day cycles, the drug produced relatively manageable side effects, which confirmed the corollary Phase I trial from Japan. ${ }^{38}$ The most prevalent adverse reactions were hematological in nature. These included grade 3-4 leucopenia and neutropenia with only $6 \%$ of patients developing grade 3 febrile neutropenia. The non-hematologic adverse reactions were rare and included mucositis, gastrointestinal toxicity, and sensory neuropathy. The sensory neuropathy was present in approximately one-third of the patients in the study but was reportedly reversible in some patients upon continuous exposure. A second, smaller Phase II multicenter study in Japan also evaluated eribulin in previously treated patients with progressive disease. The trial enrolled 51 patients, most of whom had either leiomyosarcoma or adipocytic sarcoma. The 12-week progression-free rate and PFS in the combined group of leiomyosarcoma and adipocytic sarcomas were $60 \%$ and 5.5 months, respectively. The progressionfree rate at 12 weeks in the other cohort was $31.3 \%$. The most common side effects were neutropenia, leukopenia, and lymphopenia. ${ }^{49}$

To confirm these findings, a randomized multicenter Phase III study of eribulin compared to dacarbazine in patients with leiomyosarcoma and adipocytic sarcoma was conducted. ${ }^{50}$ The study enrolled 452 patients who had already received at least two systemic treatment regimens including an anthracycline with advanced high-/intermediate-grade 
leiomyosarcoma or adipocytic sarcoma of dedifferentiated, myxoid, round cell, or pleomorphic variants. Up to grade 2 sensory neuropathy per Common Terminology Criteria for Adverse Events was allowed. Patients received eribulin at the same dose as in the Phase II trial. The dacarbazine dose was required to be prespecified by the treating physician and could be administered at $850,1,000$, or $1,200 \mathrm{mg} / \mathrm{m}^{2}$ every 21 days. The primary end point of the study was OS with PFS as a secondary end point. The study also explored other secondary end points of progression-free rate at 12 weeks, safety/tolerability, objective response rate, and health-related quality of life.

Median OS was found to be 13.5 versus 11.5 months in the eribulin and dacarbazine arms, respectively $(\mathrm{HR}=0.768$, $95 \%$ CI $=0.618-0.954 ; P=0.0169)$. The OS was longer than predicted in both study arms based on historical data, which had predicted a 2.5 -month OS benefit in the eribulin arm over dacarbazine. The median PFS was 2.6 months in both arms $(\mathrm{HR}=0.877,95 \% \mathrm{CI}=0.710-1.085 ; P=0.2287)$. Progression-free rate at 12 weeks between the two arms was also not significantly different: $33 \%$ in the eribulin arm and $28.6 \%$ in the dacarbazine arm (odds ratio $=1.3,95 \%$ $\mathrm{CI}=0.8-1.9 ; P=0.253$ ). The objective response rate and rate of stable disease were similar between the two groups: $3.9 \%$ and $52.2 \%$ and $4.9 \%$ and $47.8 \%$ for the eribulin and dacarbazine arms, respectively. After stratification, median OS in patients with adipocytic sarcoma was 15.6 versus 8.4 months in eribulin and dacarbazine, respectively (HR $=0.511,95 \%$ CI $=0.346-0.753)$. In leiomyosarcoma, however, the median OS was 12.7 versus 13 months in the eribulin and dacarbazine arms, respectively $(\mathrm{HR}=0.927$, 95\% CI $=0.714-1.203$ ). Post-study treatments (eg, chemotherapy, surgery, radiotherapy) were fairly comparable between the two study arms. In the eribulin arm, $69.3 \%$ of patients received post-study therapy versus $62.9 \%$ in the dacarbazine arm. Naturally, the major difference between the two populations was that more patients in the eribulin arm received post-study dacarbazine (34.2\% versus $7.6 \%)$. In the dacarbazine arm, $2.7 \%$ of patients received post-study eribulin. As a point of comparison, Table 1 outlines the efficacy parameters of eribulin for each Phase III trial in breast cancer, NSCLC, and STS. Additionally, Table 2 outlines the efficacy parameters and major adverse effects of eribulin in all Phase II/III trials in STS.

Overall, the authors of this study concluded that eribulin met its primary outcome in the study by significantly prolonging OS; however, with the disparity in post-study drug treatment between the two arms, it is fair to speculate whether or not post-study dacarbazine treatment in patients initially receiving eribulin may have skewed eribulin's true benefit

Table I Efficacy parameters in Phase III clinical trials of eribulin in breast cancer, non-small-cell lung cancer, and soft-tissue sarcoma

\begin{tabular}{|c|c|c|c|c|}
\hline Study & Eribulin dose & Response rate & $\begin{array}{l}\text { Median progression- } \\
\text { free survival (months) }\end{array}$ & $\begin{array}{l}\text { Overall survival } \\
\text { (months) }\end{array}$ \\
\hline \multicolumn{5}{|c|}{ Cortes et $\mathrm{al}^{39}$ : breast cancer } \\
\hline Eribulin & $1.4 \mathrm{mg} / \mathrm{m}^{2}$ on days I & $13.0 \%$ & 3.7 & 13.1 \\
\hline \multirow[t]{2}{*}{ Control (physician's choice) } & and 8 of 21 -day cycle & $5.0 \%$ & 2.2 & 10.6 \\
\hline & & $P=0.002$ & $P=0.137$ & $P=0.04 \mathrm{I}$ \\
\hline \multicolumn{5}{|c|}{ Kaufman et $\mathrm{al}^{40}$ : breast cancer } \\
\hline Eribulin & $1.4 \mathrm{mg} / \mathrm{m}^{2}$ days on I & $11.0 \%$ & 4.1 & 15.9 \\
\hline \multirow[t]{2}{*}{ Control (capecitabine) } & and 8 of 21 -day cycle & $11.5 \%$ & 4.2 & 14.5 \\
\hline & & $P=0.85$ & $P=0.30$ & $P=0.056$ \\
\hline \multicolumn{5}{|c|}{ Spigel et $\mathrm{al}^{42}$ : non-small-cell lung cancer } \\
\hline Eribulin & $1.4 \mathrm{mg} / \mathrm{m}^{2}$ on days I & $12.2 \%$ & 3.0 & 9.5 \\
\hline \multirow[t]{2}{*}{ Control (physician's choice) } & and 8 of 21 -day cycle & $15.2 \%$ & 2.8 & 9.5 \\
\hline & & NR & $P=0.395$ & $P=0.134$ \\
\hline \multicolumn{5}{|c|}{ Schoffski et $\mathrm{al}^{50}$ : soft-tissue sarcoma } \\
\hline Eribulin & $1.4 \mathrm{mg} / \mathrm{m}^{2}$ on days I & $4.0 \%$ & 2.6 & 13.5 \\
\hline \multirow[t]{2}{*}{ Control (dacarbazine) } & and 8 of 21 -day cycle & $5.0 \%$ & 2.6 & 11.5 \\
\hline & & $P=0.62$ & $P=0.23$ & $P=0.0169$ \\
\hline \multicolumn{5}{|c|}{ Schoffski et $\mathrm{al}^{50}$ : leiomyosarcoma } \\
\hline Eribulin & $1.4 \mathrm{mg} / \mathrm{m}^{2}$ on days I & NR & NR & 12.7 \\
\hline Control (dacarbazine) & and 8 of 21 -day cycle & NR & NR & 13 \\
\hline \multicolumn{5}{|c|}{ Schoffski et $\mathrm{al}^{50}$ : adipocytic sarcoma } \\
\hline Eribulin & $1.4 \mathrm{mg} / \mathrm{m}^{2}$ on days I & NR & NR & 15.6 \\
\hline Control (dacarbazine) & and 8 of 21 -day cycle & NR & NR & 8.4 \\
\hline
\end{tabular}

Abbreviation: NR, not reported. 
Table 2 Efficacy parameters and adverse effects in Phase II and Phase III clinical trials of eribulin in STS

\begin{tabular}{|c|c|c|c|c|c|}
\hline Study & Dose & $\begin{array}{l}\text { Response } \\
\text { rate }\end{array}$ & $\begin{array}{l}\text { Median progression- } \\
\text { free survival (months) }\end{array}$ & $\begin{array}{l}\text { Overall } \\
\text { survival }\end{array}$ & $\begin{array}{l}\text { Most common adverse } \\
\text { effects (grade } \geq 3 \text { ) }\end{array}$ \\
\hline \multicolumn{6}{|l|}{ Schoffski et al ${ }^{48}$} \\
\hline Leiomyosarcoma $(\mathrm{n}=38)$ & $1.4 \mathrm{mg} / \mathrm{m}^{2}$ on days I and & $0.0 \%(n=0)$ & 2.9 & NR & Neutropenia (52\%), \\
\hline Adipocytic $(n=32)$ & 8 of 21 -day cycle & $6.0 \%(n=2)$ & 2.6 & NR & leucopenia (35\%), anemia \\
\hline Synovial $(n=19)$ & & $5.0 \%(n=1)$ & 2.6 & NR & $(7 \%)$, and fatigue $(7 \%)$ \\
\hline Other STS $(n=26)$ & & $4.0 \%(n=1)$ & 2.1 & NR & \\
\hline \multicolumn{6}{|l|}{ Naito et $\mathbf{a l}^{38}$} \\
\hline Adipocytic/leiomyosarcoma $(n=52)$ & $\begin{array}{l}\mathrm{I} .4 \mathrm{mg} / \mathrm{m}^{2} \text { on days I and } \\
8 \text { of } 2 \mathrm{I} \text {-day cycle }\end{array}$ & $0.0 \%(n=0)$ & 5.5 & NR & $\begin{array}{l}\text { Neutropenia }(86 \%) \text {, } \\
\text { leucopenia }(75 \%) \text {, anemia } \\
\text { (I I.8\%), and fatigue }(7.8 \%)\end{array}$ \\
\hline \multicolumn{6}{|l|}{ Schoffski et al ${ }^{50}$} \\
\hline Adipocytic/leiomyosarcoma $(n=228)$ & $\begin{array}{l}\mathrm{I} .4 \mathrm{mg} / \mathrm{m}^{2} \text { on days I and } \\
8 \text { of } 2 \mathrm{I} \text {-day cycle }\end{array}$ & $4.0 \%(n=9)$ & 2.6 & 13.5 & $\begin{array}{l}\text { Neutropenia }(35 \%) \text {, } \\
\text { leukopenia }(10 \%) \text {, and } \\
\text { anemia }(7 \%)\end{array}$ \\
\hline
\end{tabular}

Abbreviations: STS, soft-tissue sarcoma; NR, not reported.

in these patients. In this regard, the end point of OS can be a misleading assessment tool of patients with poor prognoses and who typically (and ethically) receive post-study treatments after failing an intervention drug. Data from the Phase III trial have recently led the FDA to approve eribulin for use in patients with unresectable or metastatic liposarcoma previously treated with an anthracycline-containing regimen. The results from this Phase III trial in STS were remarkably similar to that in breast cancer, where an OS benefit was seen without a difference in PFS. There was, however, a difference in favor of eribulin in breast cancer for objective response rate. This has led investigators to hypothesize that eribulin's activity in these diseases may be due to a novel mechanism of action on tumor microenvironment, such as epithelial-to-mesenchymal transition.

\section{Safety and tolerability}

Eribulin's tolerability profile has been well documented among a multitude of patients, especially in the Phase III studies on breast cancer and STS, which have been consistent with eribulin's Phase I studies. In the EMBRACE trial, the most common adverse effects in the 270 patients taking eribulin were fatigue (54\%), neutropenia (52\%), and alopecia (45\%). Grade 3 or 4 adverse events that occurred more frequently with eribulin compared to physician's choice therapy included neutropenia (45\%), leucopenia (14\%), and peripheral neuropathy $(8 \%) \cdot{ }^{39}$ In the STS Phase III trial, the most common adverse events in the eribulin arm included neutropenia (43.8\%), fatigue (43.8\%), and nausea $40.3 \%$. Grade 3 or 4 events that occurred more commonly in the eribulin arm than in the dacarbazine arm included neutropenia $(35.4 \%)$, fatigue $(3.1 \%)$, nausea $(0.9 \%)$, constipation
$(0.9 \%)$, pyrexia $(0.9 \%)$, and peripheral sensory neuropathy $(1.8 \%)$. More patients in the dacarbazine arm experienced thrombocytopenia, anemia, and asthenia. A recent study ${ }^{8}$ demonstrating superiority of trabectedin versus dacarbazine in the management of patients with refractory STS was associated with a $37 \%$ and a $17 \%$ rate of grade 3 or 4 neutropenia and thrombocytopenia, respectively. There was also a grade 3 or 4 rate of alanine aminotransferase increase and aspartate aminotransferase increase in $26 \%$ and $13 \%$ of patients, respectively. In the Phase III eribulin trials, grade 3 or 4 peripheral neuropathy occurred at a range of $1.8 \%-8 \%$, with discontinuation of study drug in $\sim 5 \%$ of patients. Both the EMBRACE trial and the STS Phase III trial allowed for patients with preexisting neuropathy up to grade 2 and similarly found that in some patients with grade 3 or 4 peripheral neuropathy who continued treatment, there was improvement to a lower grade.

\section{Quality of life}

Quality of life of patients receiving eribulin was specifically assessed in the metastatic breast cancer trial of eribulin versus capecitabine. ${ }^{40}$ Patients in this trial were evaluated using the European Organization for Research and Treatment of Cancer Quality-of-Life Questionnaire-Core 30 questions and breast module-23 questions. Data were collected at baseline and for 2 years following initiation of treatment or until disease progression or start of a new treatment. The post hoc analysis of quality of life found that the majority of patients on eribulin maintained or improved their functioning with regard to their baseline. ${ }^{51}$ With regard to symptoms, patients taking eribulin were most affected by systemic side effects of dry mouth, different tastes, irritated eyes, hot flashes, 
feeling ill, and upset by hair loss, as opposed to those on capecitabine, who suffered more from gastrointestinal adverse effects (eg, diarrhea and vomiting). Patients in the eribulin arm also had significantly worse scores for sexual functioning and body image.

\section{Conclusion and perspectives}

Eribulin is a novel microtubule inhibitor, possessing manageable adverse effects, and FDA approved for metastatic breast cancer for patients previously treated with an anthracycline or a taxane and recently approved for patients with unresectable or metastatic liposarcoma previously treated with an anthracycline-containing regimen. The recent Phase III trial in STS demonstrated that treatment with eribulin results in a benefit in OS but not PFS for patients previously treated with an anthracycline and at least one other regimen. When stratified by histology, the benefit in OS was seen in the adipocytic sarcoma group and not the leiomyosarcoma group. The improvement in OS and not PFS is intriguing and similar to the data on breast cancer. A difference in OS without a difference in the radiographic progression of the tumor in both cancers may indicate that eribulin's effect on OS may pertain to its alteration of the tumor's microenvironment. Tubulin-binding drugs have been widely implicated in targeting tumor vasculature either by preventing neo-vascularization or by disrupting already existing tumor vasculature. ${ }^{52,53}$ It has been suggested in breast cancer xenografts that eribulin is novel in the sense that as a tubulin-binding drug, it neither disrupts preexisting vasculature nor inhibits angiogenesis but rather increases tumor perfusion. ${ }^{26}$ Altering the tumor's hypoxic microenvironment may contribute to an effect the drug may have on preventing tumor metastasis. Eribulin has also been shown to interfere with metastasis in both in vitro and in vivo experiments with regard to epithelial-mesenchymal transition. ${ }^{27}$

The lack of survival benefit in the leiomyosarcoma cohort is also of interest. A study of dacarbazine in the treatment of STS as second- or third-line therapy, demonstrated no activity in the seven patients with liposarcoma. Two patients had a partial response, and two patients had stable disease in the 14 patients with leiomyosarcoma. In fact, overall, the LMS patients comprised the two of three responses noted in study on different STS subtypes. ${ }^{12}$ By definition, more patients in the eribulin arm of the Phase III STS study received dacarbazine post-progression. Exposure to the active drug dacarbazine may have therefore masked an OS benefit in the LMS cohort of patients.

In addition to the results seen with eribulin in STS, two other relatively new agents, trabectedin and pazopanib, have recently been studied in the Phase III setting and have been FDA approved for STS in the US. Trabectedin was compared in a similar fashion to dacarbazine in patients with liposarcoma or leiomyosarcoma who received doxorubicin and at least one other prior regimen. In contrast to eribulin, however, a PFS benefit, but not OS benefit, was seen. In the PALETTE study, ${ }^{14}$ pazopanib when compared to placebo in refractory sarcoma also demonstrated a PFS but not OS benefit. The PALETTE study did not include adipocytic STS patients due to lack of efficacy in this cohort in the Phase II study; therefore, the PFS benefit with pazopanib only pertains to non-adipocytic STS. Similarly, eribulin's Phase III data do not extend to other STS subtypes as the primary end point was not met for these cohorts in the Phase II study. Because eribulin's efficacy in STS is in prolonging OS rather than PFS, taken together, these three new drugs offer different benefits for patients with different treatment goals, and different histologic STS subtypes. Though prolonging OS is considered the gold standard end point of drug trials, control of disease progression is also meaningful, as it may provide palliation of tumor-related symptoms such as shortness of breath or pain. Patients from the Phase III eribulin study in STS did undergo health-related quality of life assessments, and these data are awaited. It is also important to note that trabectedin, pazopanib, and eribulin do not generate significant shrinkage in tumor size with only a small percentage of patients in each respective study achieving, at best, a partial response: $9.9 \%$ with trabectedin, $6 \%$ with pazopanib, and $4 \%$ with eribulin. ${ }^{13,14,50}$ This is in contrast to the historical data for doxorubicin in first-line therapy for STS showing significantly more patients achieving partial responses and some with complete responses. ${ }^{9}$

Finally, in vitro data have suggested synergy of eribulin with other chemotherapeutic agents. It has been suggested that eribulin may promote vascular perfusion of tumors; therefore, using eribulin in combination with another chemotherapy agent may have synergistic effects. Because a significant portion of patients in the eribulin arm from the Phase III study received post-study drug, the OS benefit with eribulin could be partly due to an enhanced effect by subsequent chemotherapy via vascular remodeling. ${ }^{54}$ Greater perfusion of the tumor could result in greater perfusion of drugs into what typically is a relatively hypoxic environment. A Phase I study has combined gemcitabine with eribulin, and data from other novel combinations are awaited..$^{55}$

\section{Disclosure}

The authors declare no conflicts of interest in this work. 


\section{References}

1. Miller RW, Young JL Jr, Novakovic B. Childhood cancer. Cancer. 1995;75(Suppl 1):395-405.

2. Fletcher CDM, Bridge JA, Hogendoorn PCW, Mertens F. World Health Organization Classification of Tumours of Soft Tissue and Bone. 4th ed. Lyon: IARC Press; 2013.

3. Siegel RL, Miller KD, Jemal A. Cancer statistics, 2015. CA Cancer J Clin. 2015;65(1):5-29.

4. Potter DA, Glenn J, Kinsella T, et al. Patterns of recurrence in patients with high-grade soft-tissue sarcomas. J Clin Oncol. 1985;3(3): 353-366.

5. Billingsley KG, Burt ME, Jara E, et al. Pulmonary metastases from soft tissue sarcoma: analysis of patterns of diseases and postmetastasis survival. Ann Surg. 1999;229(5):602-610.

6. Italiano A, Mathoulin-Pelissier S, Cesne AL, et al. Trends in survival for patients with metastatic soft-tissue sarcoma. Cancer. 2011;117(5): 1049-1054.

7. Van Glabbeke M, Verweij J, Judson I, Nielsen OS; EORTC Soft Tissue and Bone Sarcoma Group. Progression-free rate as the principal endpoint for phase II trials in soft-tissue sarcomas. Eur J Cancer. 2002; 38(4):543-549.

8. Demetri GD, von Mehren M, Jones R, et al. Efficacy and safety of trabectedin or dacarbazine for metastatic liposarcoma or leiomyosarcoma after failure of conventional chemotherapy: results of a phase III randomized multicenter clinical trial. J Clin Oncol. 2015;62:4734. Epub 2015 Sep 14.

9. Benjamin RS, Wiernik PH, Bachur NR. Adriamycin: a new effective agent in the therapy of disseminated sarcomas. Med Pediatr Oncol. 1975; 1(1):63-76.

10. Borden EC, Amato DA, Rosenbaum C, et al. Randomized comparison of three adriamycin regimens for metastatic soft tissue sarcomas. J Clin Oncol. 1987;5(6):840-850.

11. Lorigan P, Verweij J, Papai Z, et al. Phase III trial of two investigational schedules of ifosfamide compared with standard-dose doxorubicin in advanced or metastatic soft tissue sarcoma: a European Organization for Research and Treatment of Cancer Soft Tissue and Bone Sarcoma Group study. J Clin Oncol. 2007;25(21):3144-3150.

12. Zucali PA, Bertuzzi A, Parra HJ, Campagnoli E, Quaqliuolo V, Santoro A. The "old drug" decarbazine as a second/third line chemotherapy in advanced soft tissue sarcomas. Invest New Drugs. 2008; 26(2):175-181.

13. Demetri GD, von Mehren M, Jones RL, et al. Efficacy and safety of trabectedin or dacarbazine for metastatic liposarcoma or leiomyosarcoma after failure of conventional chemotherapy: results of a phase III randomized multicenter clinical trial. J Clin Oncol. 2016;34(8):786-793.

14. van der Graaf WT, Blay JY, Chawla SP, et al. Pazopanib for metastatic soft-tissue sarcoma (PALETTE): a randomized, double-blind, placebocontrolled phase 3 trial. Lancet. 2012;379(9829):1879-1886.

15. Skubitz KM, Haddad PA. Paclitaxel and pegylated-liposomal doxorubicin are both active in angiosarcoma. Cancer. 2005;104(2):361-366.

16. Rosen G, Forscher C, Lowenbraun S, et al. Synovial sarcoma. Uniform response of metastases to high dose ifosfamide. Cancer. 1994;73(10): 2506-2511.

17. Jones RL, Fisher C, Al-Muderis O, Judson IR. Differential sensitivity of liposarcoma subtypes to chemotherapy. Eur J Cancer. 2005; 41(18):2853-2860.

18. Maki RG, Wathen JK, Patel SR, et al. Randomized phase II study of gemcitabine and docetaxel compared with gemcitabine alone in patients with metastatic soft tissue sarcomas: results of sarcoma alliance for research through collaboration study 002. J Clin Oncol. 2007; 25(19):2755-2763.

19. Seddon B, Scurr M, Jones RL, et al. A phase II trial to assess the activity of gemcitabine and docetaxel as first line chemotherapy treatment in patients with unresectable leiomyosarcoma. Clin Sarcoma Res. 2015;5:13.

20. Bramwell VH, Anderson D, Charette ML; Sarcoma Disease Site Group. Doxorubicin-based chemotherapy for the palliative treatment of adult patients with locally advanced or metastatic soft tissue sarcoma. Cochrane Database Syst Rev. 2003;(3):CD003293.
21. Judson I, Verweij J, Gelderblom H, et al. Doxorubicin alone versus intensified doxorubicin plus ifosfamide for first-line treatment of advanced or metastatic soft-tissue sarcoma: a randomized controlled phase 3 trial. Lancet Oncol. 2014;15(4):415-423.

22. Jordan MA, Kamath K, Manna T, et al. The primary antimitotic mechanism of action of the synthetic halichondrin E7389 is suppression of microtubule growth. Mol Cancer Ther. 2005;4(7):1086-1095.

23. Towle MJ, Salvato KA, Budrow J, et al. In vitro and in vivo anticancer activities of synthetic macrocyclic ketone analogues of halichondrin B. Cancer Res. 2001;61(3):1013-1021.

24. Kuznetsov G, Towle MJ, Cheng H, et al. Induction of morphological and biochemical apoptosis following prolonged mitotic blockage by halichondrin B macrocyclic ketone analog E7389. Cancer Res. 2004; 64(16):5760-5766.

25. Suzuki H, Hirata Y, Suzuki N, et al. Characterization of a new small bowel adenocarcinoma cell line and screening of anti-cancer drug against small bowel adenocarcinoma. Am J Pathol. 2015;185(2): 550-562.

26. Funahashi Y, Okamoto K, Adachi Y, et al. Eribulin mesylate reduces tumor microenvironment abnormality by vascular remodeling in preclinical human breast cancer models. Cancer Sci. 2014;105(10): 1334-1342.

27. Yoshida T, Ozawa Y, Kimura T, et al. Eribulin mesilate suppresses experimental metastasis of breast cancer cells by reversing phenotype from epithelial-mesenchymal transition (EMT) to mesenchymal-epithelial transition (MET) states. Br J Cancer. 2014;110(6):1497-1505.

28. Yu MJ, Zheng W, Seletsky BM. From micrograms to gram: scale-up synthesis of eribulin mesylate. Nat Prod Rep. 2013;30(9):1158-1164.

29. Aicher TD, Buszek KR, Fang FG, et al. Total synthesis of halichondrin B and norhalichondrin B. J Am Chem Soc. 1992;114(8):3162-3164.

30. Wozniak KM, Nomoto K, Lapidus RG, et al. Comparison of neuropathyinducing effects of eribulin mesylate, paclitaxel, and ixabepilone in mice. Cancer Res. 2011;71(11):3952-3962.

31. Swami U, Chaudhary I, Ghalib MH, Goel S. Eribulin - a review of preclinical and clinical studies. Crit Rev Oncol Hematol. 2012;81(2): 163-184.

32. Smith JA, Wilson L, Azarenko O, et al. Eribulin binds at microtubule ends to a single site on tubulin to suppress dynamic instability. Biochemistry. 2010;49(6):1331-1337.

33. Dabydeen DA, Burnett JC, Bai R, et al. Comparison of the activities of the truncated halichondrin B analog NSC 707389 (E7389) with those of the parent compound and a proposed binding site on tubulin. Mol Pharmacol. 2006;70(6):1866-1875.

34. Synold TW, Morgan RJ, Newman EM, et al. A phase I pharmacokinetic and target validation study of the novel anti-tubulin agent E7389: a California Cancer Consortium trial. J Clin Oncol. 2005;23(16 Suppl):3036.

35. Goel S, Mita AC, Mita M, et al. A phase I study of eribulin mesylate (E7389), a mechanistically novel inhibitor of microtubule dynamics, in patients with advanced solid malignancies. Clin Cancer Res. 2009; 15(12):4207-4212.

36. Tan AR, Rubin EH, Walton DC, et al. Phase I study of eribulin mesylate administered once every 21 days in patients with advanced solid tumors. Clin Cancer Res. 2009;15(12):4213-4219.

37. Vahdat LT, Pruitt B, Fabian CJ, et al. Phase II study of eribulin mesylate, a halichondrin $\mathrm{B}$ analog, in patients with metastatic breast cancer previously treated with an anthracycline and a taxane. J Clin Oncol. 2009; 27(18):2954-2961.

38. Naito Y, et al. Phase II study of eribulin mesylate in patients (pts) with advanced soft tissue sarcoma (sts). J Clin Oncol. 2014;32:10567.

39. Cortes J, O'Shaughnessy J, Loesch D, et al. Eribulin monotherapy versus treatment of physician's choice in patients with metastatic breast cancer (EMBRACE): a phase 3 open-label randomized study. Lancet. 2011;377(9769):914-923.

40. Kaufman PA, Awada A, Twelves C, et al. Phase III open-label randomized study of eribulin mesylate versus capecitabine in patients with locally advanced or metastatic breast cancer previously treated with an anthracycline and a taxane. J Clin Oncol. 2015;33(6): 594-601. 
41. Twelves C, Cortes J, Vahdat L, et al. Efficacy of eribulin in women with metastatic breast cancer: a pooled analysis of two phase 3 studies. Breast Cancer Res Treat. 2014;148(3):553-561.

42. Spigel DR, Barlesi F, Felip E, et al. Efficacy and safety of eribulin compared with treatment of physician's choice (tpc) in patients with advanced non-small-cell lung cancer (NSCLC); results from a phase III study: locally advanced non-small cell lung cancer. Int J Radiat Oncol Biol Phys. 2014;90(5):1266.

43. de Bono JS, Molife LR, Sonpavde G, et al. Phase II study of eribulin mesylate (E7389) in patients with metastatic castration-resistant prostate cancer stratified by prior taxane therapy. Ann Oncol. 2012;23(5): 1241-1249.

44. Stein MN, Chen Y, Hudes GR, Carducci MA, Tan W, DiPaola RS. A phase II study of eribulin mesylate (E7389) in patients (pts) with metastatic castration-resistant prostate cancer (crpc). J Clin Oncol. 2010;28(15 Suppl):4556.

45. Hensley ML, Kravetz S, Jia X, et al. Eribulin mesylate (halichondrin B analog E7389) in platinum-resistant and platinum-sensitive ovarian cancer: a 2-cohort, phase 2 study. Cancer. 2012;118(9):2403-2410.

46. Ikeda Y, Masashi T, Kouta H, et al. Weekly administration of bevacizumab, eribulin, and oxalilplatin in patients with platinum-resistant and refractory ovarian carcinomas: a phase II study. J Clin Oncol. 2014; 32(5 Suppl):5566.

47. Towle MJ, Nomoto K, Asano M, Kishi Y, Yu MJ, Littlefield BA. Broad spectrum preclinical antitumor activity of eribulin (Halaven $(R))$ : optimal effectiveness under intermittent dosing conditions. Anticancer Res. 2012;32(5):1611-1619.
48. Schoffski P, Ray-Coquard IL, Cioffi A, et al. Activity of eribulin mesylate in patients with soft-tissue sarcoma: a phase 2 study in four independent histological subtypes. Lancet Oncol. 2011;12(11):1045-1052.

49. Naito Y, Kawai A, Araki N, et al. Phase II study of eribulin mesylate in patients (pts) with advanced soft tissue sarcoma (sts). J Clin Oncol. 2014;32(5 Suppl):10567.

50. Schoffski P, Chawla S, Maki RG, et al. Eribulin versus dacarbazine in previously treated patients with advanced liposarcoma or leiomyosarcoma: a randomised, open-label, multicentre, phase 3 trial. Lancet. 2016;387(10028):1629-1637.

51. Cortes J, Hudgens S, Twelves C, et al. Health-related quality of life in patients with locally advanced or metastatic breast cancer treated with eribulin mesylate or capecitabine in an open-label randomized phase 3 trial. Breast Cancer Res Treat. 2015;154(3):509-520.

52. Tozer GM, Kanthou C, Baquley BC. Disrupting tumour blood vessels. Nat Rev Cancer. 2005;5(6):423-435.

53. Belotti D, Vergani V, Drudis T, et al. The microtubule-affecting drug paclitaxel has antiangiogenic activity. Clin Cancer Res. 1996;2(11): 1843-1849.

54. Young R, Woll P. Eribulin in soft-tissue sarcoma. Lancet. 2016; 387(10028):1594-1596.

55. Lheureux S, Oza AM, Laurie SA, et al. A phase I combination doseescalation study of eribulin mesylate and gemcitabine in patients with advanced solid tumours: a study of the Princess Margaret Consortium. Br J Cancer. 2015;113(11):1534-1540.
OncoTargets and Therapy

\section{Publish your work in this journal}

OncoTargets and Therapy is an international, peer-reviewed, open access journal focusing on the pathological basis of all cancers, potential targets for therapy and treatment protocols employed to improve the management of cancer patients. The journal also focuses on the impact of management programs and new therapeutic agents and protocols on

\section{Dovepress}

patient perspectives such as quality of life, adherence and satisfaction. The manuscript management system is completely online and includes a very quick and fair peer-review system, which is all easy to use. Visit http://www.dovepress.com/testimonials.php to read real quotes from published authors. 